\title{
Standard Stars: CCD Photometry, Transformations and Comparisons
}

\author{
Hwankyung Sung ${ }^{1}$ and Michael. S. Bessell ${ }^{2}$ \\ ${ }^{1}$ Bohyun-san Optical Astronomy Observatory, Yeongcheon 770-820, Korea \\ sungh@boao.re.kr \\ ${ }^{2}$ Research School of Astronomy \& Astrophysics, Australian National University, \\ Private Bag, Weston Creek PO, ACT 2611, Australia
}

Received 2000 July 13, accepted 2000 August 3

\begin{abstract}
We discuss variations of the atmospheric extinction coefficients and transformation equations to the standard UBVRI system based on observations of standard stars during 1996-1997 at Siding Spring Observatory using a thinned SITe CCD and coloured glass filters. In the transformation from the initial natural system to the Landolt version of the standard system, a large nonlinear term related to the Balmer discontinuity was required for the $U$ transformation. We then modified the $U$ filter, and the subsequent transformation to the SAAO version of the standard $U B V R I$ system had only small nonlinear correction terms for $U, B$, and $I$. The correction terms relating to $U$ and $B$ are evidently due to the Balmer discontinuity, while that relating to $I$ seems to be due to the Paschen discontinuity at $\lambda \approx 8200 \AA$. We also compared the results with Landolt's observations, and confirmed the difference between the two sets of standard stars (SAAO and Landolt).
\end{abstract}

Keywords: techniques: photometric—stars: imaging—standards

\section{Introduction}

Observations of standard stars are of crucial importance in stellar photometry. Currently two main sets of standard stars are used for astronomical broadband $U B V R I$ photometry. One is the E-region standards centred at declination $-45^{\circ}$ which provide fundamental standards for $U B V R I$ photometry in the southern hemisphere. These regions were developed to calibrate photographic magnitudes where the north polar sequence was not observable. The establishment of the E-region standard star photometry was performed by Dr A. W. J. Cousins. Astronomers at the South African Astronomical Observatory (SAAO) continue to refine the accuracy (Menzies et al. 1989) and to extend the colour range (Kilkenny et al. 1998) of the standards. Although E-regions provide the most accurate standards available currently, there are several limitations. E-regions are available only in the southern hemisphere, and many E-region standards are too bright and too sparsely distributed, and therefore not well suited for modern CCD observations.

The other main set is the Landolt standards (Landolt $1973,1983,1992)$ along the celestial equator. Initially the system comprised only $U B V$, but has since been extended to Cousins' RI. Landolt standards have several strong points. The first is their availability from both hemispheres. The second is the inclusion of several blue and red stars in a small area, which makes it easier to obtain the transformation coefficients. Third, the magnitude coverage was extended to faint stars to provide standard stars for CCDs on large telescopes.

Cousins (1984a,b) found some small systematic differences between his photometry and that of Landolt for some stars in common in the equatorial regions. Later, Menzies et al. (1991) performed extensive photometry of Landolt equatorial standards and demonstrated the extent and nature of the systematic differences with respect to the E-region standards. Bessell (1995) derived transformation relations between the SAAO system [and the Bessell (1990b) extension for the reddest stars] and the Landolt system.

Both systems were established with photoelectric photometers, while most current photometry is undertaken using CCDs. In most cases, linear transformations to the standard system are employed for the CCD photometry. Linear transformations are possible when the combined responses of filter and detector for the two photometric systems are very similar. The bandpasses of the wideband $U B V R I$ system are relatively well defined (see Bessell 1990a) as are reasonable glass filter combinations. The quantum efficiencies of CCD chips are also usually similar across the $V, R$ and $I$ bands but can vary greatly in the UV and blue due to different processing steps in CCD manufacture. Some CCDs have zero UV and low blue quantum efficiency (QE) because they are 'thick' CCDs. Others are thinned, but the surface treatment and anti-reflection coatings differ, resulting in different $\mathrm{UV}$ and blue responses. Thick (and thin) CCDs can also be coated with a fluorescent material such as Lumogen that absorbs UV and blue photons and converts them into green ones better detected by the CCD. As a result of all these effects, the combined response of filter and detector can produce drastically different $U$ and $B$ bands for different CCDs. The existence of discontinuities in stellar energy distributions or the presence of emission lines may then give rise to nonlinear terms in the transformation equations.

In this work, we will discuss the transformation of CCD photometry to the standard systems based on observations of standard stars made during observing runs at Siding Spring Observatory (SSO) in 1996 and 1997. In 1996 August, November and 1997 January, 
February/March we observed Landolt standard regions. In 1997 May/June, August and November, SAAO Eregions (Menzies et al. 1989), SAAO measurements of equatorial standards (Menzies et al. 1991) and blue and red standards (Kilkenny et al. 1998) were observed. In Section 2, we will discuss the atmospheric extinction coefficients. The transformation relations will be dealt with in Section 3. Some comparisons will be made in the same section. The results will be summarised in Section 4.

\section{Atmospheric Extinction}

\subsection{SSO CCD System}

All the observational materials were obtained at SSO with the 40 inch telescope $(f / 8)$ and a thinned SITe 2048 $\times 2048 \mathrm{CCD}(24 \mu \mathrm{m}$ pixels). Across the $U$ passband, the SITe CCD shows a rapid change in sensitivity $(\mathrm{QE} \approx$ $60 \%$ at $\lambda=4000 \AA$ and $10 \%$ at $\lambda=3000 \AA$ ). The specifications of the filters used in the observations are summarised in Table 1.

Table 1. Filter specification

\begin{tabular}{|c|c|}
\hline Filter & Combination \\
\hline$\overline{\mathrm{U}}$ & $\begin{array}{l}\text { UG1 } 1 \mathrm{~mm}+\mathrm{S} 86122 \mathrm{~mm} \\
+ \text { WG295 } 2 \mathrm{~mm}(+\mathrm{UG} 11 \mathrm{~mm})^{\mathrm{a}}\end{array}$ \\
\hline B & BG37 $3 \mathrm{~mm}+$ GG395 $1 \mathrm{~mm}+$ BG39 $1 \mathrm{~mm}$ \\
\hline $\mathrm{V}$ & GG495 $2 \mathrm{~mm}+\mathrm{BG} 403 \mathrm{~mm}$ \\
\hline $\mathrm{R}$ & KG3 2 mm + OG570 3 mm \\
\hline I & RG9 $2 \mathrm{~mm}+\mathrm{WG} 2953 \mathrm{~mm}$ \\
\hline
\end{tabular}

${ }^{a}$ From 1997 May/Jun observing run

The response function of the $U$ filter used in the CCD photometry is similar to the combined response of the $U$ filter and 1P21 photomultiplier tube of the original Johnson $U$, but the steeply sloping QE variation across the $U$ passband results in an overall CCD $U$ band response function significantly different from the standard $U$ passband. Sung et al. (1998) included a nonlinear correction term $\left(f\left[(B-V)_{0}\right]\right)$ in the $U$ transformation to the Landolt standards to account for the Balmer Jump, as well as a linear term in $U-B$ to correct for the effective wavelength shift. The transformation equation they used is

$$
\begin{aligned}
U= & u-\left[k_{1 U}-0.013(U-B)\right] X \\
& \left.+0.102(U-B)+f[B-V)_{0}\right]+\zeta_{U},
\end{aligned}
$$

where $U,(U-B)$ and $(B-V)$ denote standard values, $u$, $(u-b)$ and $(b-v)$ are instrumental values, $k_{1 U}$ is the primary extinction coefficient for $U, X$ is the airmass and $\zeta_{U}$ is the zero point in $U$. From 1997 May, for the $U$ observations we added an additional $1 \mathrm{~mm}$ UG1 filter to the existing $U$ filter in order to shift the effective wavelength toward the UV and closer to that of standard $U$. In addition, to quantify what part of the nonlinear correction was due to the Landolt $U-B$ system (see Menzies et al. 1991; Cousins 1984a,b; see also Bessell 1995), we observed the SAAO E-regions. The numbers of usable data points for the determination of the atmospheric extinction coefficients are given in Table 2; the coefficients are given in Figure 1 below.

\subsection{Extinction Coefficients}

Atmospheric extinction is caused by the scattering or absorption of light by molecules or other particles. Most of the extinction in the visual window is due to Rayleigh scattering by air molecules. Another important contributor to the extinction is scattering and absorption by small

Table 2. Observation log

\begin{tabular}{llllll}
\hline Date of obs. & Standard stars & \multicolumn{4}{c}{ Number of data points } \\
& & $I$ & $V$ & $B$ & $U$ \\
\hline 1996.8 .25 & Mark A, SA 110, T Phe & 52 & 46 & 35 & 41 \\
1996.11 .6 & SA 98, T Phe & 27 & 51 & 59 & 37 \\
1997.1 .8 & SA 98 & 28 & 43 & 51 & 33 \\
1997.2 .28 & SA 98, PG 1323-086 & 41 & 36 & 33 & 25 \\
1997.3 .2 & PG 0918+029 & 10 & 10 & 10 & 10 \\
1997.3 .3 & SA 98, PG 1323-086 & 48 & 42 & 56 & 45 \\
\hline 1997.5 .31 & E5, E7 & 18 & 19 & 22 & 23 \\
1997.6 .1 & E5, E7 & 14 & 17 & 20 & 23 \\
1997.6 .5 & E5 & 6 & 9 & 9 & 9 \\
1997.6 .23 & E5, E7 & 13 & 16 & 17 & 18 \\
1997.6 .25 & E5, E7 & 12 & 16 & 14 & 14 \\
1997.8 .7 & SA 114, E1, HD 188112, CD -38 222 & 14 & 13 & 15 & 15 \\
1997.8 .10 & E6, E7, SA 114, CD -38 222, GL 27.1 & 22 & 28 & 26 & 26 \\
1997.11 .19 & E1, SA 93, SA 99, SA 114, CD -31 4800 & 28 & 28 & 30 & 26 \\
1997.11 .20 & SA 93, SA 99, SA 114 & 26 & 26 & 24 & 18 \\
1997.11 .23 & SA 93, SA 99, SA 114, CD -31 4800 & 16 & 16 & 23 & 21 \\
\hline
\end{tabular}




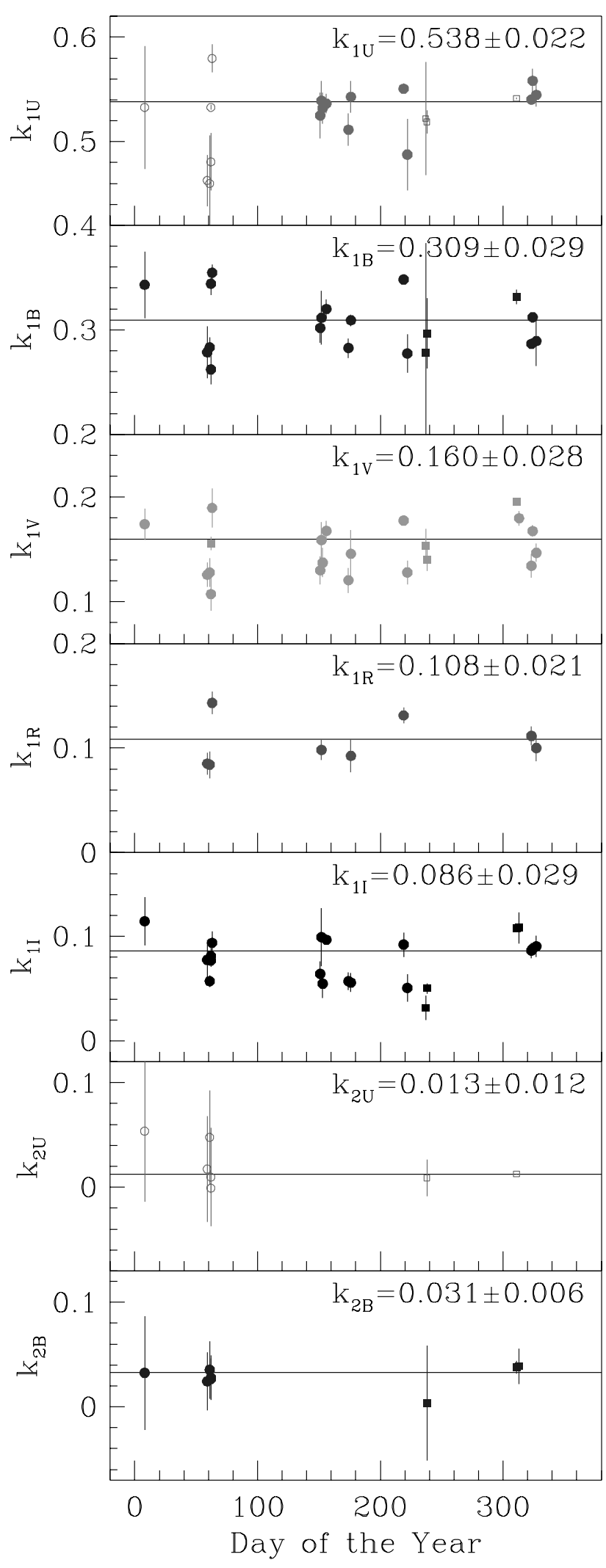

Figure 1-Measured Siding Spring extinction coefficients plotted against yearly day number. The weighted mean values are indicated by the line and the value listed in the figure. The square and circle represents, respectively, the data obtained in 1996 and 1997. The open and filled symbols in $k_{1 U}$ and $k_{2 U}$ denote data obtained without and with the additional $1 \mathrm{~mm}$ UG1 filter, respectively. liquid or solid particles of various sizes called aerosols (Cousins \& Caldwell 1998). In the case of an observatory in an urban area or at a low-altitude non-urban site, extinction by aerosols is the most important source although the constituent aerosols will differ. Volcanic eruptions and massive forest fires, for example, can inject aerosols into the stratosphere, resulting in large, widespread and long-lasting increases in extinction. Changes in the thickness of the ozone layer in the upper atmosphere also affect the extinction in the UV.

As the atmospheric extinction coefficients change mainly with changing aerosol content, in the absence of volcanic eruptions, dust storms or fires etc., the extinction coefficients are generally quite stable and the use of mean extinction coefficients is recommended as long as the standard stars and program objects are measured interspersed and at similar zenith distances (ZDs). That is, if standards and targets have similar ZD values (i.e. differ by less than 0.1 ), deviations from mean extinction (say by $0.1 \mathrm{mag}$, an unusually large difference) produce only a second-order effect (less than $0.01 \mathrm{mag}$ ) on the accuracy of the photometry. However, if the difference in airmass between standard stars and targets is large (say 0.5), then a $0.1 \mathrm{mag}$ difference between the true extinction and the mean extinction will result in a difference of 0.05 mag in the photometry, an unacceptable error. We determined atmospheric extinction coefficients under photometric conditions. By observing standard stars from the meridian to $z \approx 60^{\circ}$ (i.e. airmass $\approx 2$ ), we obtained a long baseline in airmass and thus measured precise extinction. In $V, R$ and $I$, there was no evidence for secondary extinction coefficients $\left(k_{2}\right)$ so only the primary extinction coefficients $\left(k_{1}\right)$ were derived. In $U$ and $B$, however, secondary extinction coefficients were evident. We determined and used weighted mean values of secondary coefficients for the atmospheric extinction corrections.

We plot the derived extinction coefficients obtained in 1996-97 in Figure 1. Usually we observed only UBVI and therefore only a few $k_{1 R}$ values are shown. The large scatter in the secondary extinction coefficients was caused mainly by a lack of blue stars in the observed regions. The weighted mean values (weight $=1 / \varepsilon^{2}$, where $\varepsilon$ is the standard error of the coefficient) are marked in the figure.

The fluctuations in $k_{1}$ are up to \pm 0.05 for a given filter. The use of an additional UG1 $1 \mathrm{~mm}$ filter in $U$ observation did not appear to cause any noticeable difference in the $U$ extinction. The mean values of the primary extinction coefficients are very similar to those obtained by Landolt at Cerro Tololo (see Table 1 of Landolt 1992). Only $I$ showed a seasonal variation in extinction, being lower in winter. On 1997 August 9, the first third of the night showed normal extinction; it was non-photometric in the second part and the last part yielded a small value of $k_{1 I}$. Probably variations in the column density of $\mathrm{H}_{2} \mathrm{O}$ (strong absorption bands of $\mathrm{H}_{2} \mathrm{O}$ fall within the $I$ band) caused the variations in the $I$ extinction coefficient. 

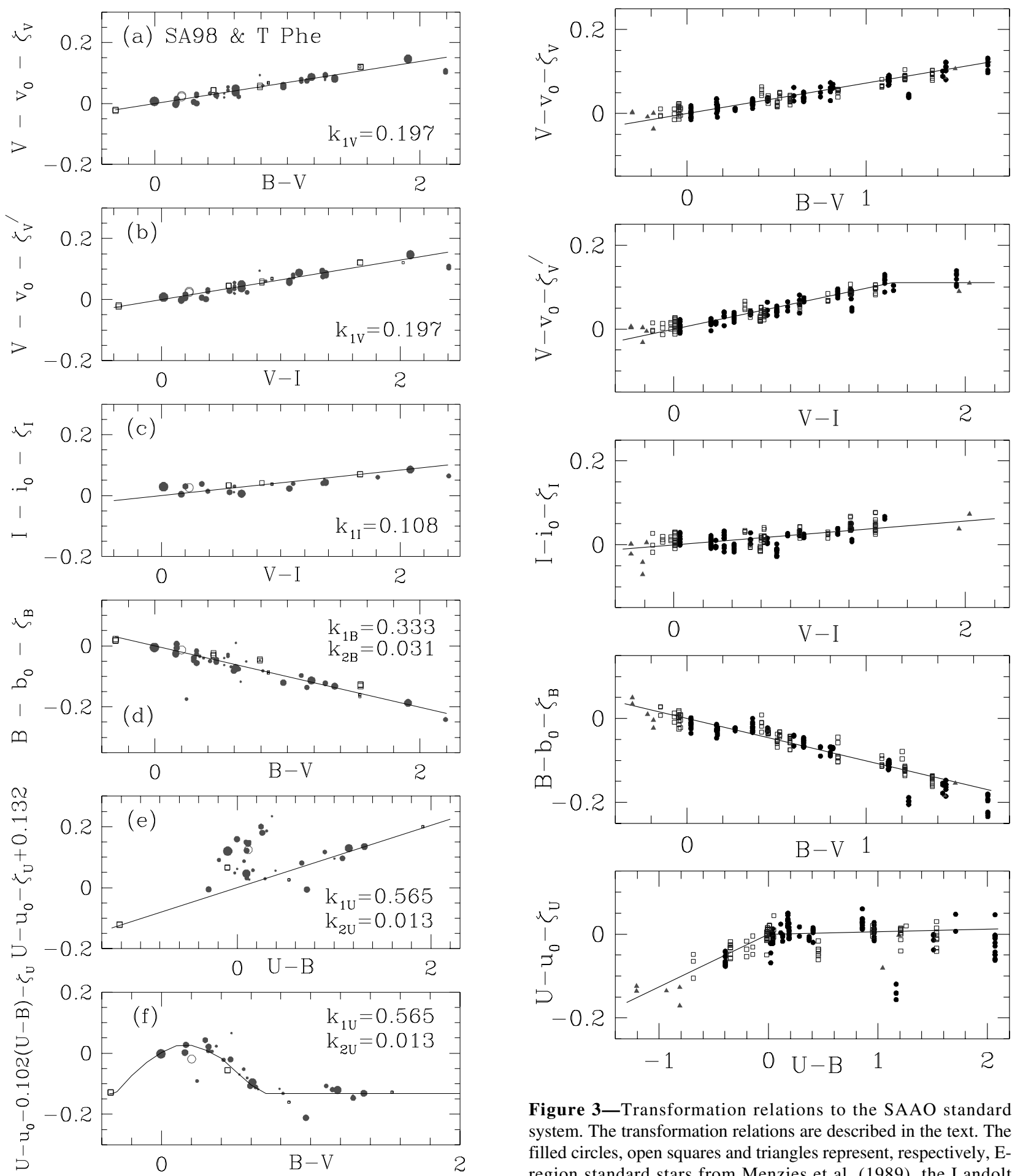

Figure 2-A typical sample of transformation relations to the Landolt standard system (1996 November 6). The transformation relations can be found in Sung et al. (1998). The squares, filled circles, and open circles represent, respectively, stars in the T Phe region, SA 98 region, and SA 98-185 (an Algol-type eclipsing binary; Kim, Sung \& Lee 1997). The sizes of the symbols are proportional to the numbers of independent photoelectric observations in Landolt (1992). The extinction coefficients used for the extinction correction are given in the figure.
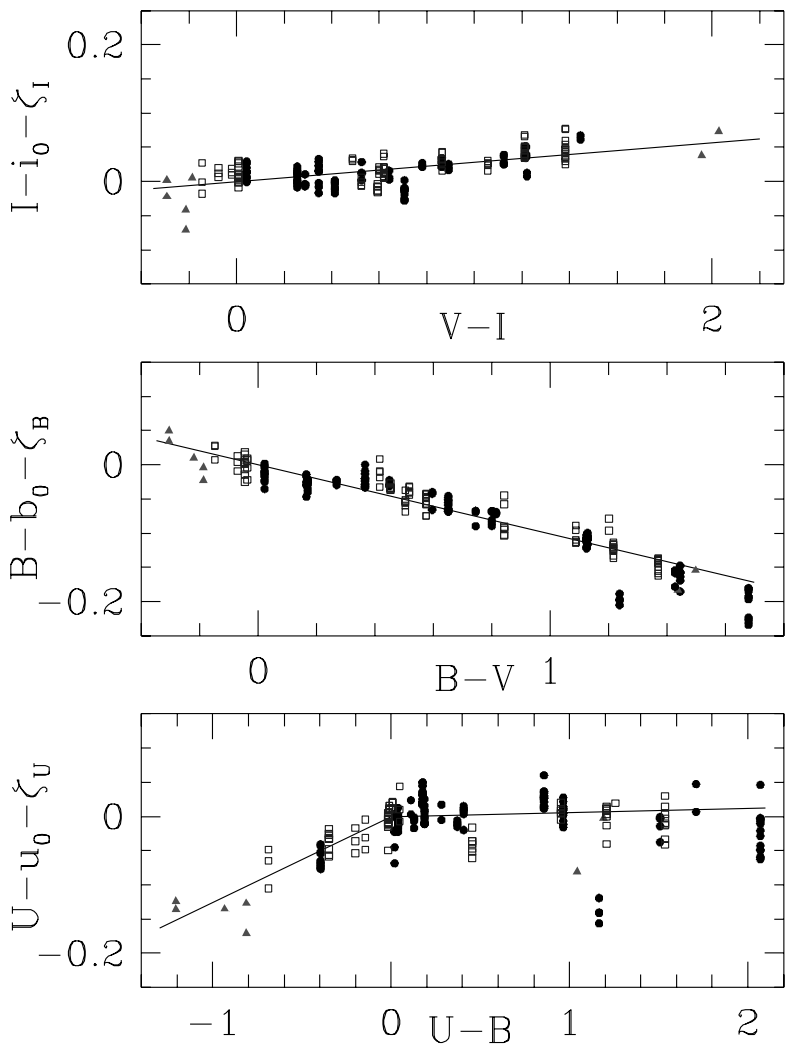

Figure 3-Transformation relations to the SAAO standard system. The transformation relations are described in the text. The filled circles, open squares and triangles represent, respectively, Eregion standard stars from Menzies et al. (1989), the Landolt equatorial standard stars from Menzies et al. (1991) and blue and red standard stars from Kilkenny et al. (1998). The uppercase characters, lowercase characters with subscript 0 , and $\zeta$ denote standard magnitudes, atmospheric-extinction-corrected instrumental magnitudes, and zero point, respectively. The thin line represents the adopted transformation relation for each band.

\section{Standard System Transformations}

\subsection{Landolt Standards}

After bias subtraction and flat-field division, we performed simple aperture photometry on the standard star 
frames. An aperture of $14^{\prime \prime}$ diameter was used in the standard star photometry to simulate the photoelectric photometry done by Landolt (1992). In deriving the transformation coefficients, we used only stars with good signal-to-noise ratios ( $\varepsilon \leq 0.01 \mathrm{mag}$ ). In addition, the reliability of the standard star values was also very important. We used stars with $n_{p e} \geq 5$ only and assigned a weight for each star based on $n_{p e}$. Final values of the transformation coefficients were determined by averaging the individual determinations. The resulting transformation coefficients can be found in Sung et al. (1998) and a typical case is shown in Figure 2. In the vertical axis labelling, the uppercase characters, lowercase characters with subscript 0 , and $\zeta$ represent standard magnitudes, atmospheric-extinction-corrected instrumental magnitudes and zero point, respectively.

There is no evidence for a change in slope in the $V$ transformation up to $V-I=2$, but for the SAAO standard stars, the slope changed at $V-I=1.5$ (see Figure 3). For the $I$ transformation, there is a weak indication of a slope change at $V-I=0.7$. Menzies (1993) outlined the nonlinearities involved in the standardisation of the current natural photoelectric UBVRI system at the SAAO. Linear transformations to $V$ have a break at $B-V=1.5$; those to $V-I$ have a break at $V-I$ $=1.6$; and those to $B-V$ have a break at 1.0 and again at 1.6. The neglect of such breaks has lead to some of the systematic differences between different versions of the $U B V R I$ system.

In Figure 2e, we see that a simple linear transformation is not suitable for $U$. A large scatter in $U$ can be seen at $U-B \sim 0$. This suggests that the scatter is related to the Balmer discontinuity and the confluence of the Balmer lines. The linear transformation coefficient for the $U$ magnitude against $U-B$ is the slope derived simply from two stars with extreme $U-B$ colours in the $\mathrm{T}$ Phe region. The large value of the slope indicates that the effective wavelength of the $U$ band is much too far to the red. After applying this linear $U-B$ correction term, the residuals were plotted against $(B-V)\left[(B-V)_{0}\right.$ for T Phe $C$ ] as shown in Figure $2 f$. The thin line represents the nonlinear correction term $f\left[(B-V)_{0}\right]$ in the $U$ transformation. The size of the nonlinear correction is at most about 0.13 mag.

Part of this correction arises from the rapid change in the UV sensitivity of the thinned SITe $2048 \times 2048$ CCD shifting the $U$ passband redward so that the stellar fluxes above the Balmer discontinuity contribute too much to $U$, and part of the correction also arises from the $U-B$ of Landolt standard stars [three stars in SA 98 showed up to 0.06 mag difference relative to the measurements of Menzies et al. (1991)]. We will discuss this further in Section 3.3.

Several standard regions observed in the 1997 January, February/March observing runs (Ru 149, PG $0918+029$, and PG 1323 -086) are well described by the transformation relation found in Figure 2e,f. However, stars in the SA 110 region observed in 1996
August do not show evidence of the nonlinear term in the $U$ transformation. This probably results from the fact that SA 110 is near the Galactic Plane, and many stars may be strongly affected by interstellar reddening and follow a different correction curve.

\subsection{SAAO Standards}

3.2.1 Transformation Coefficients. The problems with the $U$ transformation to the Landolt standard $U$ system encouraged us to observe the SAAO standard stars and also to shift the effective wavelength of the $U$ band further to the UV and thus diminish the contribution of the light above the Balmer jump. To do this we added an additional $1 \mathrm{~mm}$ of UG1 to the existing $U$ filter. This unfortunately also had the effect of lowering the overall throughput of the $U$ band, requiring an increase in exposure times.

One difficulty in standardising CCD photometry is that there are only a few standard stars on a CCD image. The lack of stars having extreme colours is another difficulty in determining transformation coefficients accurately. It is clear that efforts are still required to provide more and better CCD standard fields. We included the equatorial stars measured by Menzies et al. (1991) and blue and red standard stars observed by Kilkenny et al. (1998). We averaged the individual determinations to derive the final transformation coefficients. The final transformation relations are:

$$
\begin{aligned}
V= & V-k_{1 V} X+0.072(B-V)+\varsigma_{V} \\
V= & v-k_{1 V} X+0.074(V-I)+\varsigma^{\prime} V,(V-I \leq 1.5) \\
V= & V-k_{1 V} X+\varsigma^{\prime \prime} V,(V-I>1.5) \\
B= & b-\left[k_{1 B}+0.031(B-V)\right] X-0.101(B-V)+\varsigma_{B} \\
U= & u-\left[k_{1 U}-0.013(U-B)\right] X+0.125(U-B) \\
& +\varsigma_{U},(U-B \leq 0.0) \\
U= & u-\left[k_{1 U} X-0.013(U-B)\right] X+0.006(U-B) \\
& +\varsigma_{U},(U-B>0.0) \\
I= & i-k_{1 I} X+0.028(V-I)+\varsigma_{I} .
\end{aligned}
$$

In the $V$ transformation with respect to the $V-I$ colour, there is evidently no dependence on $V-I$ for red stars $(V-I>1.5)$. This is probably related to the fact that $V$ photometric standards were originally set up using $B-V$ as the colour term. The $B-V$ colour of late$\mathrm{K}$ and $\mathrm{M}$ stars shows little variation while $V-I$ continues to increase to later spectral types. As mentioned above, Landolt standard stars do not show evidence of a slope change up to $V-I=2.0$. The transformation of $U$ is also very different from that found previously (see Section 3.1). Due to the use of an additional $1 \mathrm{~mm}$ UG1 filter, which suppresses the contribution of the Balmer discontinuity, nearly no $U-B$ dependence for late-type stars was found. Somewhat large scatters in the figure are caused mainly by the mixed use of data obtained on different nights.

3.2.2 Residuals. We plot in Figure 4 the residuals relative to the SAAO system after transforming to the 

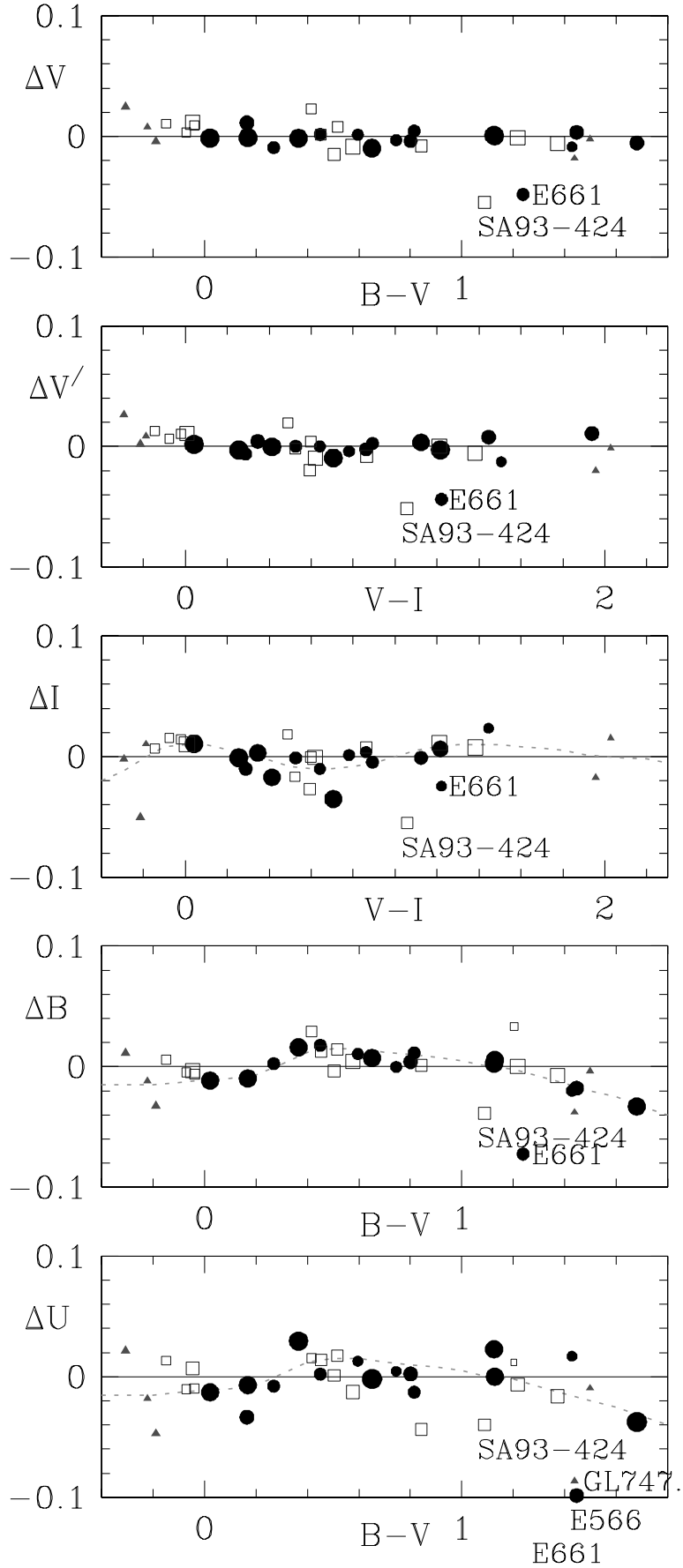

Figure 4-The average of residuals relative to the SAAO system plotted against standard colour. The $\Delta$ represents the difference between the standard and observed magnitudes. The sizes of symbols are proportional to the numbers of observations. All the symbols are the same as in Figure 3. The dotted lines represent the mean systematic differences found from the data.

standard system using the relations above. The blue and red stars in Kilkenny et al. (1998) show slightly larger scatter. This is probably not caused by the photometric errors, but by errors in the extinction correction for the stars having extreme colour. For $V$, there is no evidence of a systematic difference relative to the standard system, but for $I, B$ and $U$ there exist small but systematic
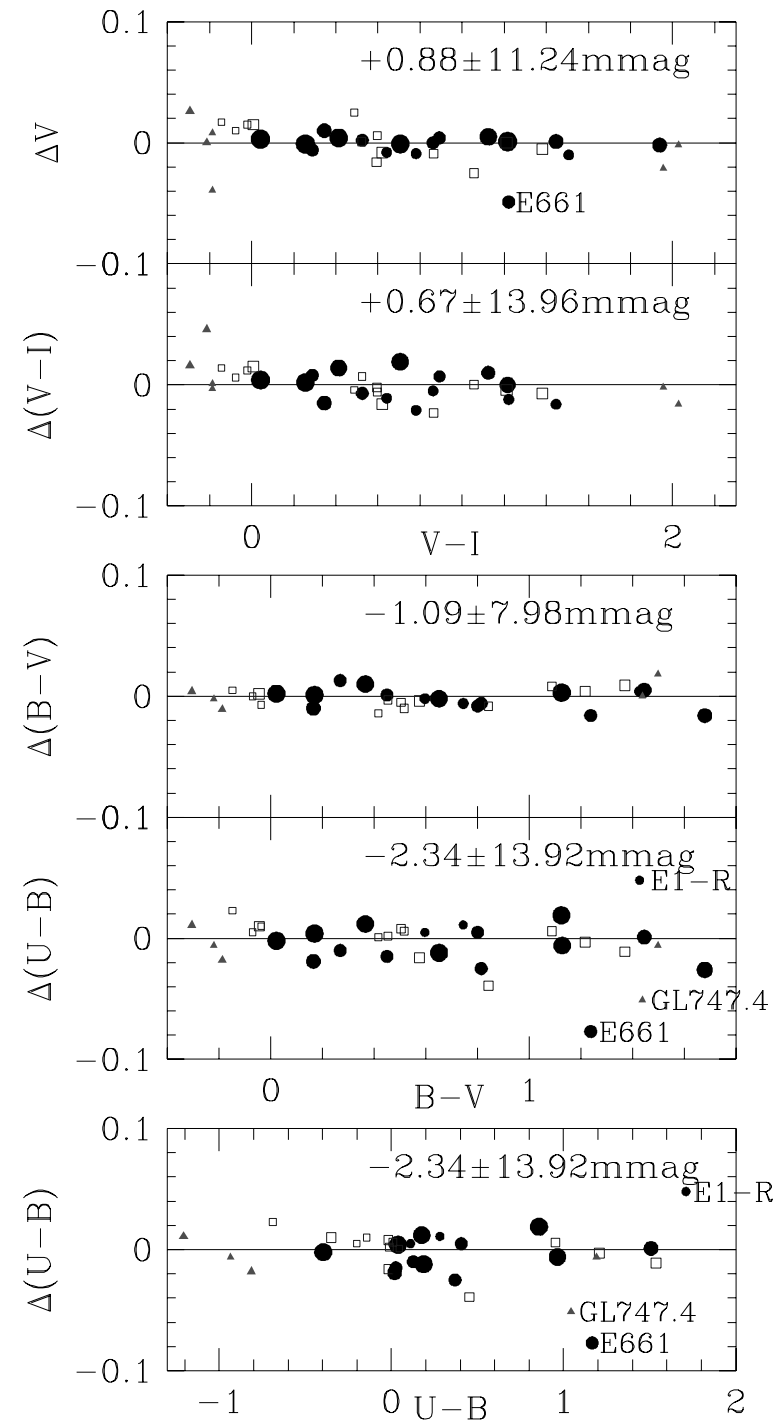

Figure 5-Difference between Table 3 a values and the original SAAO photometry. The symbols used are the same as in Figure 2. The zero point difference and its scatter (s.d.) are marked in each panel. Three deviant stars are shown. The sense of $\Delta$ is photoelectric magnitude minus CCD magnitude.

differences. Evidently the systematic differences in $U$ and $B$ are related to the Balmer discontinuity. The maximum difference at $B-V=0.5$ is about $0.02 \mathrm{mag}$. The difference seems to increase for red stars, but the lack of very red stars (due to a lack of highly reddened stars among the standard stars) makes it difficult to confirm this proposition.

Even though the scatter is large, we can be confident that systematic differences exist in $I$. Observations from individual nights show clearly the change of slope at $V-$ $I \approx 0.7$. The large scatter is probably caused by the difficulty in the determination of accurate zero points due to the lack of stars of extreme colour, and by variations in atmospheric water vapour content that affect fluxes in the $I$ band more than those in other bands. The systematic differences in $I$ are almost certainly caused by the fact that the standard $I$ passband is defined on the 
Table 3a. Photometric data: standard stars

\begin{tabular}{|c|c|c|c|c|c|c|c|c|c|c|c|c|}
\hline Star & $V$ & $V-I$ & $B-V$ & $U-B$ & $\varepsilon_{V}$ & $\varepsilon_{V-I}$ & $\varepsilon_{B-V}$ & $\varepsilon_{U-B}$ & & & $n_{\mathrm{obs}}$ & \\
\hline E1-R & 9.474 & $\ldots$ & 1.424 & 1.662 & 0.006 & & 0.006 & 0.003 & 2 & & 2 & 1 \\
\hline E1-O & 9.764 & 0.652 & 0.598 & 0.106 & 0.007 & 0.001 & 0.005 & 0.001 & 2 & 2 & 2 & 1 \\
\hline E1-Q & 9.861 & 0.802 & 0.751 & 0.271 & 0.005 & 0.002 & 0.005 & 0.001 & 2 & 2 & 2 & 1 \\
\hline E525 & 9.372 & 0.867 & 0.809 & 0.401 & 0.005 & 0.009 & 0.010 & 0.011 & 4 & 2 & 4 & 4 \\
\hline E544 & 9.968 & 0.360 & 0.175 & 0.038 & 0.007 & 0.007 & 0.004 & 0.012 & 6 & 6 & 6 & 6 \\
\hline E566 & 9.873 & 1.462 & 1.441 & 1.507 & 0.012 & 0.013 & 0.010 & 0.011 & 6 & 2 & 6 & 6 \\
\hline E567 & 10.200 & 1.138 & 1.116 & 0.925 & 0.014 & 0.011 & 0.012 & 0.006 & 6 & 5 & 6 & 6 \\
\hline E5-R & 9.331 & $\ldots$ & 1.696 & 2.096 & 0.015 & 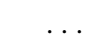 & 0.008 & 0.010 & 6 & & 6 & 8 \\
\hline E5-O & 9.996 & 0.399 & 0.356 & 0.164 & 0.005 & 0.007 & 0.008 & 0.007 & 12 & 9 & 10 & 10 \\
\hline E5-T & 10.825 & 0.687 & 0.653 & 0.199 & 0.007 & 0.007 & 0.009 & 0.009 & 12 & 10 & 10 & 11 \\
\hline E661 & 10.199 & 1.233 & 1.254 & 1.243 & 0.003 & 0.002 & 0.004 & 0.011 & 4 & 2 & 4 & 4 \\
\hline E6-U & 9.956 & 0.533 & 0.449 & 0.041 & 0.003 & 0.010 & 0.003 & 0.003 & 4 & 4 & 4 & 4 \\
\hline E6-W & 10.538 & 0.280 & 0.256 & 0.138 & 0.002 & 0.001 & 0.001 & 0.004 & 4 & 4 & 4 & 4 \\
\hline E6-Y & 10.150 & 0.885 & 0.821 & 0.395 & 0.004 & 0.005 & 0.004 & 0.003 & 4 & 3 & 4 & 4 \\
\hline E737 & 9.783 & 1.114 & 1.125 & 0.971 & 0.008 & 0.008 & 0.006 & 0.015 & 10 & 5 & 10 & 10 \\
\hline E7-W & 10.549 & 0.253 & 0.168 & 0.034 & 0.004 & 0.008 & 0.005 & 0.015 & 13 & 12 & 11 & 11 \\
\hline E7-X & 10.773 & 0.038 & 0.020 & -0.393 & 0.009 & 0.011 & 0.005 & 0.010 & 13 & 12 & 11 & 11 \\
\hline E7-c & 10.405 & 1.216 & 1.122 & 0.838 & 0.004 & 0.005 & 0.005 & 0.013 & 13 & 8 & 11 & 11 \\
\hline SA93-317 & 11.558 & 0.596 & 0.509 & -0.025 & 0.004 & 0.003 & 0.008 & 0.008 & 7 & 6 & 6 & 7 \\
\hline SA93-326 & 9.573 & 0.517 & 0.456 & -0.012 & 0.006 & 0.009 & 0.004 & 0.003 & 5 & 4 & 5 & 5 \\
\hline SA93-332 & 9.791 & 0.604 & 0.527 & 0.002 & 0.005 & 0.009 & 0.008 & 0.009 & 5 & 5 & 5 & 5 \\
\hline SA93-333 & 12.032 & 0.887 & 0.851 & 0.493 & 0.003 & 0.010 & 0.011 & 0.018 & 7 & 6 & 6 & 7 \\
\hline SA93-424 & 11.640 & 1.057 & 1.082 & 0.947 & 0.007 & 0.006 & 0.009 & 0.018 & 6 & 5 & 5 & 6 \\
\hline SA93-417 & 11.942 & 0.816 & 0.755 & 0.280 & 0.003 & 0.007 & 0.010 & 0.013 & 7 & 6 & 6 & 7 \\
\hline SA93-407 & 11.972 & 0.889 & 0.884 & 0.618 & 0.004 & 0.006 & 0.015 & 0.018 & 7 & 6 & 6 & 7 \\
\hline SA93-405 & 12.212 & 0.598 & 0.509 & -0.012 & 0.004 & 0.007 & 0.012 & 0.016 & 7 & 6 & 6 & 7 \\
\hline SA93-312 & 12.035 & 0.667 & 0.598 & 0.054 & 0.008 & 0.009 & 0.016 & 0.006 & 6 & 5 & 5 & 5 \\
\hline SA93-422 & 12.143 & 0.745 & 0.608 & 0.063 & 0.005 & 0.006 & 0.011 & 0.021 & 4 & 3 & 3 & 4 \\
\hline SA99-408 & 9.802 & 0.492 & 0.430 & 0.046 & 0.009 & 0.010 & 0.004 & 0.007 & 4 & 3 & 4 & 3 \\
\hline SA99-418 & 9.452 & -0.032 & -0.031 & -0.154 & 0.012 & 0.011 & 0.004 & 0.005 & 3 & 3 & 3 & 3 \\
\hline SA99-438 & 9.390 & -0.158 & -0.154 & -0.711 & 0.007 & 0.013 & 0.002 & 0.005 & 3 & 3 & 3 & 3 \\
\hline SA99-447 & 9.418 & -0.082 & -0.070 & -0.205 & 0.005 & 0.008 & 0.004 & 0.005 & 3 & 3 & 3 & 3 \\
\hline SA114-750 & 11.915 & -0.007 & -0.047 & -0.359 & 0.011 & 0.015 & 0.004 & 0.009 & 12 & 12 & 11 & 9 \\
\hline SA114-755 & 10.918 & 0.635 & 0.580 & 0.000 & 0.005 & 0.012 & 0.005 & 0.005 & 12 & 12 & 11 & 9 \\
\hline SA114-670 & 11.117 & 1.215 & 1.213 & 1.212 & 0.007 & 0.013 & 0.006 & 0.011 & 12 & 12 & 11 & 9 \\
\hline SA114-548 & 11.609 & 1.389 & 1.362 & 1.547 & 0.005 & 0.015 & 0.006 & 0.016 & 12 & 12 & 11 & 9 \\
\hline SA114-654 & 11.853 & 0.706 & 0.660 & 0.205 & 0.002 & 0.017 & 0.002 & 0.007 & 6 & 6 & 5 & 5 \\
\hline SA114-651 & 10.272 & 0.686 & 0.612 & 0.070 & 0.001 & 0.001 & 0.001 & 0.001 & 1 & 1 & 1 & 1 \\
\hline CD-38222 & 10.462 & -0.184 & -0.219 & -0.928 & 0.001 & 0.001 & 0.001 & 0.002 & 1 & 1 & 1 & 1 \\
\hline GL27.1 & 11.406 & 2.044 & 1.480 & 1.198 & 0.001 & 0.001 & 0.001 & 0.006 & 1 & 1 & 1 & 1 \\
\hline GL747.4 & 11.320 & 1.958 & 1.438 & 1.094 & 0.001 & 0.001 & 0.002 & 0.005 & 1 & 1 & 1 & 1 \\
\hline HD188112 & 10.196 & -0.260 & -0.177 & -0.795 & 0.018 & 0.003 & 0.007 & 0.005 & 2 & 2 & 2 & 2 \\
\hline CD-31 4800 & 10.524 & -0.309 & -0.310 & -1.218 & 0.002 & 0.013 & 0.007 & 0.001 & 2 & 2 & 2 & 2 \\
\hline
\end{tabular}


Table 3b. Photometric data: additional stars

\begin{tabular}{|c|c|c|c|c|c|c|c|c|c|c|c|c|}
\hline Star & $V$ & $V-I$ & $B-V$ & $U-B$ & $\varepsilon_{V}$ & $\varepsilon_{V-I}$ & $\varepsilon_{B-V}$ & $\varepsilon_{U-B}$ & & & obs & \\
\hline E5-Y & 12.875 & 0.037 & 0.041 & 0.032 & 0.012 & 0.013 & 0.010 & 0.009 & 18 & 16 & 15 & 14 \\
\hline E5-c & 13.426 & 0.956 & 0.900 & 0.445 & 0.019 & 0.010 & 0.006 & 0.004 & 18 & 16 & 16 & 15 \\
\hline E5-h & 14.280 & 1.308 & 1.239 & 1.111 & 0.014 & 0.014 & 0.014 & 0.199 & 18 & 16 & 16 & 15 \\
\hline E5-b & 13.573 & 0.717 & 0.668 & 0.181 & 0.009 & 0.010 & 0.006 & 0.006 & 12 & 11 & 11 & 11 \\
\hline $\mathrm{E} 5-\mathrm{X}^{\mathrm{a}}$ & 12.027 & 0.812 & 0.738 & 0.283 & 0.005 & 0.009 & 0.001 & 0.009 & 14 & 11 & 12 & 11 \\
\hline E5-W & 12.053 & 0.488 & 0.412 & 0.083 & 0.005 & 0.008 & 0.004 & 0.012 & 17 & 13 & 14 & 13 \\
\hline E5-Z & 12.367 & 0.833 & 0.778 & 0.413 & 0.003 & 0.003 & 0.003 & 0.007 & 2 & 2 & 2 & 1 \\
\hline E5-a & 13.104 & 0.689 & 0.590 & 0.065 & 0.009 & 0.005 & 0.012 & 0.006 & 18 & 16 & 16 & 13 \\
\hline E5-d & 13.635 & 0.770 & 0.693 & 0.178 & 0.012 & 0.007 & 0.012 & 0.013 & 14 & 12 & 12 & 11 \\
\hline E5-e & 13.892 & 0.705 & 0.677 & 0.169 & 0.017 & 0.023 & 0.016 & 0.006 & 12 & 11 & 11 & 11 \\
\hline E5-f & 14.039 & 0.891 & 0.816 & 0.378 & 0.014 & 0.009 & 0.007 & 0.019 & 18 & 16 & 16 & 14 \\
\hline E5-g & 14.656 & 0.750 & 0.578 & -0.074 & 0.007 & 0.009 & 0.006 & 0.029 & 10 & 9 & 9 & 8 \\
\hline E6-S & 10.153 & 0.173 & 0.041 & -0.563 & 0.003 & 0.001 & 0.003 & 0.000 & 2 & 2 & 2 & 2 \\
\hline E6-a & 10.989 & 0.574 & 0.500 & 0.069 & 0.003 & 0.005 & 0.004 & 0.007 & 4 & 4 & 4 & 4 \\
\hline E6-c & 10.672 & 1.658 & 1.523 & 1.800 & 0.003 & 0.002 & 0.003 & 0.022 & 4 & 2 & 4 & 4 \\
\hline E6-d & 11.756 & 0.625 & 0.541 & 0.140 & 0.007 & 0.002 & 0.004 & 0.009 & 4 & 4 & 4 & 4 \\
\hline E6-e & 11.996 & 0.664 & 0.574 & 0.037 & 0.006 & 0.008 & 0.008 & 0.009 & 4 & 4 & 4 & 4 \\
\hline E6-f & 11.590 & 1.169 & 1.106 & 0.790 & 0.005 & 0.008 & 0.003 & 0.011 & 4 & 4 & 4 & 4 \\
\hline E6-n & 12.868 & 1.141 & 1.046 & 0.751 & 0.008 & 0.014 & 0.007 & 0.028 & 4 & 3 & 4 & 4 \\
\hline E6-p & 13.615 & 0.765 & 0.689 & 0.229 & 0.024 & 0.024 & 0.040 & 0.034 & 4 & 4 & 4 & 4 \\
\hline E6-r & 14.374 & 1.290 & 1.185 & 1.034 & 0.006 & 0.003 & 0.004 & 0.083 & 2 & 2 & 2 & 2 \\
\hline E6-s & 14.618 & 0.813 & 0.792 & 0.162 & 0.041 & 0.049 & 0.047 & 0.132 & 4 & 4 & 4 & 4 \\
\hline E6-t & 14.701 & 0.822 & 0.757 & 0.416 & 0.013 & 0.029 & 0.008 & 0.044 & 2 & 2 & 2 & 2 \\
\hline E721 & 10.137 & 0.453 & 0.408 & 0.176 & 0.013 & 0.007 & 0.010 & 0.006 & 9 & 8 & 5 & 5 \\
\hline E7-Y & 10.826 & 0.201 & 0.131 & -0.003 & 0.005 & 0.010 & 0.005 & 0.011 & 13 & 12 & 11 & 11 \\
\hline E7-a & 10.961 & 0.560 & 0.485 & 0.029 & 0.010 & 0.007 & 0.005 & 0.008 & 13 & 12 & 11 & 11 \\
\hline E7-b & 10.963 & 0.861 & 0.646 & 0.403 & 0.004 & 0.010 & 0.005 & 0.013 & 13 & 12 & 11 & 11 \\
\hline E7-d & 11.159 & 0.567 & 0.461 & 0.311 & 0.005 & 0.008 & 0.006 & 0.012 & 12 & 11 & 10 & 10 \\
\hline E7-e & 11.984 & 0.187 & 0.113 & -0.087 & 0.012 & 0.010 & 0.011 & 0.015 & 9 & 8 & 7 & 7 \\
\hline E7-f & 12.047 & 0.281 & 0.228 & 0.192 & 0.011 & 0.021 & 0.009 & 0.013 & 13 & 12 & 11 & 11 \\
\hline E7-h & 11.913 & 1.034 & 0.817 & 0.532 & 0.016 & 0.021 & 0.011 & 0.015 & 13 & 12 & 10 & 8 \\
\hline E7-i & 12.604 & 0.574 & 0.445 & 0.284 & 0.013 & 0.013 & 0.016 & 0.011 & 13 & 12 & 11 & 11 \\
\hline E7-k & 12.936 & 0.389 & 0.331 & 0.247 & 0.023 & 0.017 & 0.011 & 0.019 & 10 & 9 & 8 & 8 \\
\hline E7-1 & 12.500 & 1.232 & 1.220 & 1.159 & 0.016 & 0.010 & 0.011 & 0.041 & 13 & 12 & 11 & 11 \\
\hline E7-m & 12.537 & 1.295 & 1.306 & 1.385 & 0.016 & 0.009 & 0.009 & 0.051 & 8 & 8 & 7 & 7 \\
\hline E7-n & 13.026 & 1.261 & 1.168 & 0.889 & 0.020 & 0.015 & 0.016 & 0.046 & 12 & 11 & 10 & 10 \\
\hline E7-o & 12.844 & 1.546 & 1.536 & 1.642 & 0.015 & 0.007 & 0.023 & 0.104 & 7 & 6 & 6 & 6 \\
\hline E7-p & 13.226 & 1.292 & 1.107 & 0.652 & 0.017 & 0.022 & 0.019 & 0.058 & 12 & 11 & 10 & 10 \\
\hline E7-q & 13.053 & 1.851 & 1.662 & 1.950 & 0.010 & 0.014 & 0.032 & 0.206 & 13 & 12 & 11 & 11 \\
\hline E7-g & 12.069 & 0.566 & 0.478 & 0.058 & 0.005 & 0.006 & 0.005 & 0.012 & 13 & 12 & 11 & 11 \\
\hline E7-r & 13.406 & 1.697 & 1.623 & 1.805 & 0.014 & 0.010 & 0.023 & 0.198 & 13 & 12 & 11 & 11 \\
\hline E7-s & 14.281 & 0.753 & 0.686 & 0.203 & 0.032 & 0.049 & 0.039 & 0.071 & 13 & 12 & 11 & 11 \\
\hline
\end{tabular}

${ }^{\mathrm{a}}$ Optical double 
red side by the abrupt cutoff in the GaAs response at about $9000 \AA$, whereas the red edge of our CCD I passband is defined by the gradual cutoff of the CCD to longer wavelengths. As a result the contribution of the Paschen discontinuity $(\lambda \approx 8200 \AA)$ to the $I$ magnitude will be different for the two passbands.

3.2.3 Final Results. The final results, after systematic difference corrections are made to $U, B$ and $I$, are listed in Table 3a,b. $U-B$ is little affected by the systematic corrections to $U$ and $B$ because they mainly cancel each other out. We also list the photometric data for several stars brighter than $V=15$ in the observed fields. In Table 4 we give the mean differences between our Table 3 a values and the original SAAO data; differences for the individual stars against appropriate colours are plotted in Figure 5.

No systematic differences are now evident. In particular, there are no systematic differences between the three sets, although the stars from Kilkenny et al. (1998) show a somewhat larger scatter. That is, as mentioned above, solely due to these stars having extreme colours. The relatively large scatter in $V-I$ is caused by the difficulty in determining the zero point with too few stars of extreme colour, and by variations in absorption from atmospheric water vapour. To minimise the difference relative to the SAAO system, we should use a multi-line transformation equation for $I$.

A few stars (E1-R, GL747.4 and E661) showed large differences in $U-B$. For two of them (E1-R, GL 747.4) the SAAO data are probably at fault, as indicated by the quoted standard error in $U-B$ (for example, for GL 747.4, $\left.U=13.794, \sigma_{U-B}=0.031\right)$. For E661, the magnitude as well as the colours showed large differences $(\Delta V=-0.049, \Delta(V-I)-0.012, \Delta(B-V)=-0.016$, and $\Delta(U-B)=-0.077)$. E661 may be a variable or spectroscopically peculiar star.

Our results show that CCD photometry can achieve better than 0.015 mag accuracy. To improve the accuracy, several well-observed standard regions with many standard stars covering wide colour and magnitude ranges are necessary.

\subsection{Comparison}

3.3.1 Landolt. Cousins (1984b) showed the difference between the SAAO E-region standard system and Landolt's equatorial standard system for the first time. Later, Menzies et al. (1991) confirmed the difference from extensive photometry of Landolt's equatorial standard stars. Bessell (1995) derived the transformation relation between SAAO measurements and Landolt's measurements in a polynomial form. We observed a few standard regions along the celestial equator to extend the colour range. We also measured a few stars that were not measured by Menzies et al. (1991) but were measured by Landolt. The differences in magnitude and colour for stars in common with Landolt (1973, 1983, 1992) were also listed in Table 4 and are compared in Figure 6. The differences with respect to Landolt's measurements are consistent with those found by Menzies et al. (1991) and Bessell (1995).

The $U$ band mismatch between our initial natural and the standard systems, shown in Figure 2e,f, highlights the effects that such mismatches can have. It is likely that a bandpass mismatch exacerbated by a transformation methodology is responsible for many of the differences between the Landolt and SAAO $U-B$ systems.

The practice of transforming natural $u-b$ colours to standard $U-B$ using a colour term in $U-B$ (as done by Landolt) can result in a $U-B$ system dependent on spectral type, reddening and luminosity for some stars. This is because a colour term in $U-B$ implies that all stars with a given $U-B$ will have the same correction to the standard system, which is not necessarily true. Stars with very different spectra, such as late B, A or F stars or reddened $\mathrm{B}$ and $\mathrm{A}$ stars, can have the same standard $U-B$ value but, because of a passband mismatch, will have different natural $u-b$ colours. These differences can be partially corrected for by using a colour term in $B$ $-V$ but it is obvious that if this is not done, the resultant system will have within it systematic differences for late $\mathrm{B}, \mathrm{A}$ and $\mathrm{F}$ stars. However, even were this done, reddened stars with different $U-B$ versus $B-V$ loci to

Table 4. Comparison with photoelectric photometry

\begin{tabular}{llcll}
\hline Source & \multicolumn{1}{c}{$\Delta V^{*}$} & $\Delta(V-I)^{*}$ & $\Delta(B-V)^{*}$ & \multicolumn{1}{c}{$\Delta(U-B)^{*}$} \\
\hline Menzies et al. (1989) & $-0.44 \pm 5.51\left(16^{\mathrm{a}}\right)$ & $-1.53 \pm 12.00(15)$ & $-1.41 \pm 8.12(17)$ & $-3.87 \pm 13.73\left(15^{\mathrm{b}}\right)$ \\
Menzies et al. (1991) & $+2.00 \pm 14.43(13)$ & $+0.31 \pm 11.38(13)$ & $-1.77 \pm 7.12(13)$ & $+0.15 \pm 15.28(13)$ \\
Kilkenny et al. (1998) & $+2.20 \pm 17.04(5)$ & $+8.20 \pm 24.00(5)$ & $-1.80 \pm 10.59(5)$ & $-4.75 \pm 11.93\left(4^{\mathrm{c}}\right)$ \\
SAAO (total) & $+0.88 \pm 11.24\left(34^{\mathrm{a}}\right)$ & $+0.67 \pm 13.96(33)$ & $-1.09 \pm 7.98(35)$ & $-2.34 \pm 13.92\left(32^{\mathrm{d}}\right)$ \\
& & $\ldots$ & $-6.05 \pm 8.54(20)$ & $-12.45 \pm 15.46(20)$ \\
Landolt (1973) & $-10.00 \pm 16.58(20)$ & $\ldots .90 .913(13)$ & $-7.31 \pm 9.90(13)$ & $-14.92 \pm 25.47(13)$ \\
Landolt (1983) & $+6.31 \pm 13.97(13)$ & $+5.00 \pm 9.81(13)$ & $-7.30 \pm 12.51(10)$ & $-16.40 \pm 26.95(10)$ \\
Landolt (1992) & $-10.60 \pm 13.10(10)$ & $+6.00 \pm 9.42(10)$ & $-6.77 \pm 9.81(43)$ & $-14.12 \pm 21.26(43)$ \\
Landolt (total) & $-9.02 \pm 14.84(43)$ & $+5.43 \pm 9.44(43)$ & &
\end{tabular}

\footnotetext{
* In mmag

${ }^{\mathrm{a}}$ E661 excluded; ${ }^{\mathrm{b}}$ E1-R and E661 excluded; ${ }^{\mathrm{c}}$ GL 747.4 excluded; ${ }^{\mathrm{d}}$ E1-R, E661 and GL 747.4 excluded
} 

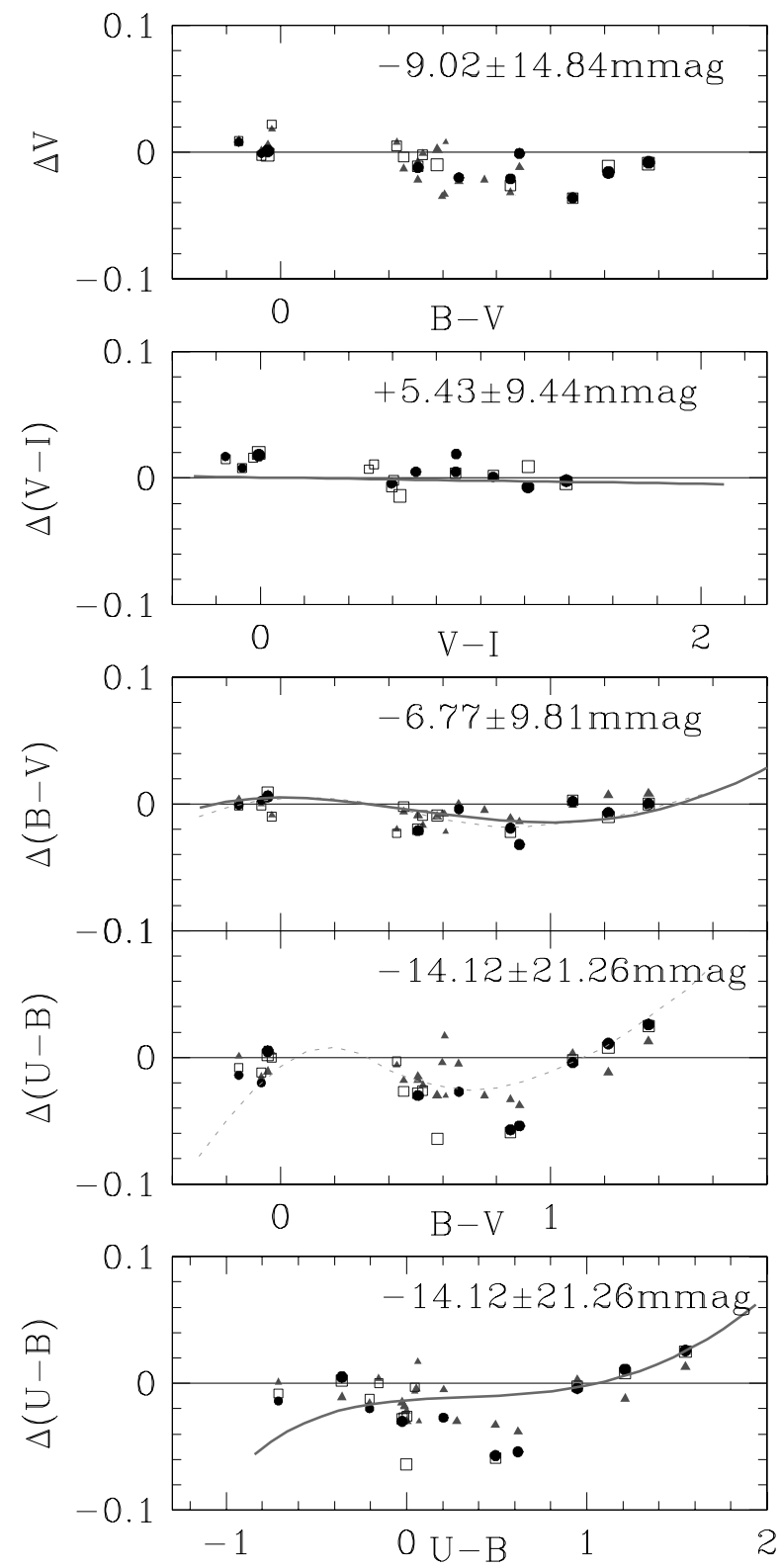

Figure 6-Comparison with Landolt's photometry. Triangles, open squares and filled circles represent, respectively, photoelectric data from Landolt (1973), Landolt (1983) and Landolt (1992). The dotted lines in the figure denote the mean differences between SAAO measurements and Landolt's measurements, from Menzies et al. (1991). The thick curved line represents the polynomial curve fitted by Bessell (1995).

those of normal stars will not follow the same regression against $B-V$ and so will not follow the same correction curve.

Landolt's natural GaAs $u-b$ system is not very close to the standard $U-B$ system and so it is likely that, because the transformations were made using a $U-$ $B$ colour term alone, systematic $U-B$ differences exist for some kinds of stars namely, A-F stars, reddened B-A stars and giant A-F stars. The effect of reddening appears more pronounced in Landolt's stars because he usefully provided many fainter stars in his lists, ensuring

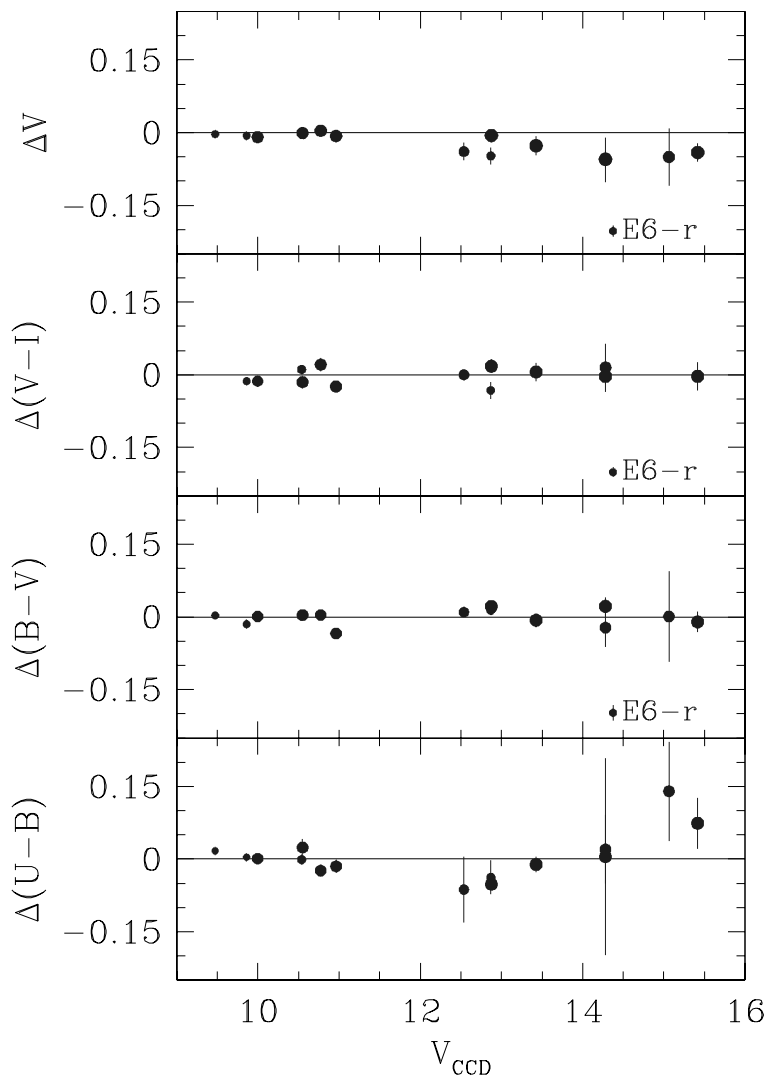

Figure 7-Comparison with Graham's photometry. The size of a symbol is proportional to the number of measurements.

a higher proportion of reddened stars compared to other lists of brighter standard stars.

It is worth noting that the difference in $U-B$ increased from Landolt's earlier work (Landolt 1973) through his more recent work (Landolt 1992). This can be understood if the early 1P21 passbands were closer to the standard passbands.

3.3.2 Graham. Graham (1982) observed many Eregion stars. The purpose of his observations was to extend the magnitude range of E-region stars. He included several faint E-region stars up to $V \approx 17$. We measured many E-region stars in the vicinity of SAAO standard stars (Table $3 b$ ). Our results are compared with Graham's data in Figure 7. The meaning of $\Delta$ is the same as before. For fainter stars $(V>12)$, Graham's $V$ magnitudes are systematically brighter than ours. $V-I$ and $B-V$, on the other hand, do not show any systematic differences relative to ours. However, $U-B$ shows a large scatter for fainter stars. This is probably caused by the poor signal-to-noise ratio of these stars in the $U$ passband. Our photometry, as well as Graham's, was affected by the low $\mathrm{S} / \mathrm{N}$ ratio in $U$. One star, E6-r, shows a large difference in magnitude as well as colour.

\section{Summary}

Based on the observational data obtained during 19961997 observing runs at SSO, we present the atmospheric 
extinction coefficients and transformation relations to the standard systems (SAAO and Landolt standard stars). The results are summarised as follows.

(1) The atmospheric extinction coefficients fluctuated by up to $\pm 0.05 \mathrm{mag}$ around the mean value for a given passband. If the difference in airmass between standard stars and target objects is small, the mean extinction coefficients give accurate results. However, if the difference is large, accurate values of the extinction coefficients, as well as of the zero points, are necessary to achieve reliable photometry.

(2) In the transformation to Landolt's standard $U$ system using our initial $U$ filter, a nonlinear term related to the Balmer discontinuity was required. While most of this correction is due to the non-standard initial $U$ passband of the CCD system, part is related to the differences in $U-B$ between Landolt's system and the SAAO system.

(3) For the SAAO standard system with a revised $U$ filter, a small but systematic nonlinear correction is required for $U, B$, and $I$. The nonlinear correction terms for $U$ and $B$ are related to the Balmer discontinuity, while that for $I$ may be related to the Paschen discontinuity at $\lambda \approx 8200 \AA$. The maximum value of the nonlinear correction is about $0.02 \mathrm{mag}$.

(4) After making the nonlinear corrections, we achieved better than 0.004 mag difference relative to the SAAO system in zero point, and obtained better than 0.015 mag in accuracy relative to the original SAAO standard value.

(5) From a direct comparison with Landolt's measurements of equatorial standard stars, we reconfirmed the mostly small but systematic differences between the two standard systems (SAAO and Landolt).
(6) Graham's observations of faint stars in E-regions show no systematic difference in colours, but the $V$ magnitudes of Graham's faint stars $(V>12)$ are systematically brighter by about $0.05 \mathrm{mag}$.

\section{Acknowledgments}

H.S. acknowledges partial support through the PostDoctoral Program of his former institution, Kyungpook National University. H.S. would also like to express his thanks to Dr S.-L. Kim for helpful comments.

\section{References}

Bessell, M. S. 1990a, PASP, 102, 1181

Bessell, M. S. 1990b, A\&AS, 83, 357

Bessell, M. S. 1995, PASP, 107, 672

Cousins, A. W. J. 1984a, South Africa Astron. Observatory Circ., No. 8,59

Cousins, A. W. J. 1984b, South Africa Astron. Observatory Circ., No. 8,69

Cousins, A. W. J., \& Caldwell, J. A. R. 1998, Observatory, 118, 85

Graham, J. A. 1982, PASP, 94, 244

Kilkenny, D., van Wyk, F., Roberts, G., Marang, F., \& Cooper, D. 1998, MNRAS, 294, 93

Kim, S.-L., Sung, H., \& Lee, S.-G. 1997, Inf. Bull. Var. Stars, No. 4466

Landolt, A. U. 1973, AJ, 78, 959

Landolt, A. U. 1983, AJ, 88, 439

Landolt, A. U. 1992, AJ, 104, 340

Menzies, J. W. 1993, in Precision Photometry, ed. D. Kilkenny, E. Lastovica \& J. W. Menzies (Cape Town: SAAO), p. 35

Menzies, J. W., Cousins, A. W. J., Banfield, R. M., \& Laing, J. D. 1989, South Africa Astron. Obs. Circ., No. 13, 1

Menzies, J. W., Marang, F., Laing, J. D., Coulson, I. M., \& Engelbrecht, C. A. 1991, MNRAS, 248, 642

Sung, H., Bessell, M. S., \& Lee, S.-W. 1998, AJ, 115, 734 